\title{
Preface for the Joint Workshop on Tools for Program Development and Analysis in Computational Science and Software Engineering for Large-Scale Computing
}

\author{
Andreas Knüpfer ${ }^{1}$, Arndt Bode ${ }^{2}$, Dieter Kranzlmüller ${ }^{3}$, Daniel Rodrìguez ${ }^{4}$, \\ Roberto Ruiz ${ }^{5}$, Jie Tao $^{6}$, Roland Wismüller ${ }^{7}$, and Jens Volkert ${ }^{8}$ \\ ${ }^{1}$ Center for Information Services and High Performance Computing \\ Technische Universität Dresden, Germany \\ ${ }^{2}$ Lehrstuhl für Rechnertechnik und Rechnerorganisation \\ Technische Universität München, Germany \\ ${ }^{3}$ Ludwig-Maximilians-Universität München (LMU) \& \\ Leibniz Supercomputing Centre (LRZ), Munich, Germany \\ ${ }^{4}$ University of Alcalá, Spain \\ ${ }^{5}$ Pablo de Olavide University of Seville, Spain \\ ${ }^{6}$ Steinbuch Center for Computing \\ Karlsruhe Institute of Technology, Germany \\ ${ }^{7}$ Operating Systems and Distributed Systems \\ University of Siegen, Germany \\ ${ }^{8}$ Institute of Graphics and Parallel Processing \\ Johannes Kepler University Linz, Austria
}

Today, computers and computational methods are increasingly important and powerful tools for science and engineering. Yet, using them effectively and efficiently requires both, expert knowledge of the respective application domain as well as solid experience applying the technologies. Only the combination allows new and faster advancement in the area of application. The same is true for establishing new computational concepts as regular methods in the field of application. This applies to either quantitative improvement (e.g. by parallel scalability) or by qualitative progress (e.g. by better algorithms).

Of course, scientists and engineers are most interested in solving the actual task. At the same time, computational tools require some knowledge about their usage and its implications. Yet, the tools must not demand intimate skills using the tool nor specialized computer science knowledge. Otherwise, the costs of tool usage and training will outweigh the benefits and it will not attract a broad user community.

The same applies in the area software engineering. The research about software engineering is being influenced by computational applications and vice versa. In one direction, software engineering methods, processes, metrics, management, etc. need to consider the way these types of applications are developed and executed. In the other direction, computational techniques can help to improve the accuracy and control of all types of projects. 
Our workshop addresses tools and methods provided by computer scientists for scientists and engineers from their respective application domains. This includes the following:

- Software development tools

- Testing and debugging tools

- Program analysis and visualization tools

- Performance analysis and tuning tools

- Management of large amounts of data and data mining

- Software development processes

- Computational intelligence techniques applied to software engineering

- Data mining software engineering repositories

- Resource management, load balancing, job queuing and accounting

- Problem solving environments for specific application domains

- Use cases and practical experiences with real-world applications

Furthermore, it covers reports about use cases and success stories using the computational tools for science and engineering by either the users or by the computer scientists or by collaboration of both.

The primary intention of this workshop is to bring together developers of tools for scientific computing and their potential users. Since its beginning at the first ICCS in 2001, the workshop has encouraged tool developers and users from the scientific and engineering community to exchange their experiences. Tool developers present to users how their tools support scientists and engineers during program development and analysis. Tool users report their experiences employing such tools, especially highlighting the benefits as well as the desired improvements. 